\title{
Cuestiones socioambientales como articulador curricular en la formación de profesores de ciencias: aproximación a un estado del arte
}

\author{
Esperanza Sepúlveda Rojas ${ }^{10}$ Universidad Distrital Francisco José de Caldas, Colombia
}

Autor de correspondencia: ${ }^{1}$ esepulvedar@correo.udistrital.edu.co Recibido: 05 de septiembre de 2020 Revisado: 12 octubre de 2020 Aprobado: 25 de marzo de 2021 Publicado: 06 de junio de 2021

\section{Resumen}

En este artículo se presenta una aproximación al estado del arte de una investigación doctoral en la línea de Inclusión de la Dimensión Ambiental en la Educación en Ciencias - Énfasis en Educación en Ciencias del DIE-UD. Para su desarrollo, se tomaron constructos de la concepción filosófica hermenéutica desde un fundamento abductivo. La metodología consistió en la lectura, análisis, interpretación, correlación y clasificación de la información. Según el grado de interés y la necesidad frente al tema, se empleó el análisis de contenido para el tratamiento de los datos, el cual fue asistido por el software Atlas.ti 7, con lo cual se estableció una ruta de reflexión crítica que permitió hacer un diagnóstico del avance del conocimiento en cuestiones socioambientales y su articulación con la formación del profesorado de ciencias. Como resultado se establecieron cuatro categorías nodales emergentes, que dan cuenta de la situación actual del campo ambiental en la educación en ciencias, como elemento contextualizador cultural, político, ético y crítico, para enfrentar las diversas situaciones de crisis socioambiental.

Palabras clave: cuestiones socioambientales, educación en ciencias, educación ambiental, formación de profesores, estado del arte

Para citar este artículo: Sepúlveda, E., \& Mora, W. (2021). Cuestiones socioambientales como articulador curricular en la formación de profesores de ciencias: aproximación a un estado del arte. Praxis \& Saber, 12(31), e12648. https://doi.org/10.19053/22160159.v12.n31.2021.12648 


\title{
Socio-environmental issues as a curricular articulator in science teachers' training: an approach to a state of the art
}

\begin{abstract}
This article presents an approach to the state of the art of a doctoral research in the line of Inclusion of the Environmental Dimension in Science Education - Emphasis on Science Education of the DIE-UD. For this purpose, constructs from the philosophical hermeneutic conception were taken based on an abductive foundation. The methodology consisted of reading, analyzing, interpreting, correlating, and classifying the information. According to the degree of interest and need regarding the topic, content analysis was used to process data, which was assisted by the Atlas.ti 7 software. A critical reflection route was established that made it possible to diagnose the progress of knowledge on socio-environmental issues and their articulation with science teachers' training. As a result, four emerging nodal categories were established, which reflect the current situation of the environmental field in science education, as a cultural, political, ethical, and critical contextualizing element, in order to face different situations of socio-environmental crisis.
\end{abstract}

Keywords: socio-environmental issues, science education, environmental education, teacher training, state of the art

\section{As questões socioambientais como um articulador curricular na formação de professores de ciências: uma abordagem ao estado da arte}

\section{Resumo}

Este artigo apresenta uma abordagem ao estado da arte de uma pesquisa de doutorado na linha de Inclusão da Dimensão Ambiental na Educação em Ciências - Ênfase na Educação em Ciências do DIE-UD. Para este fim, os constructos da concepção filosófica hermenêutica foram tomados de um fundamento abdutivo. A metodologia consistiu na leitura, análise, interpretação, correlação e classificação das informações. De acordo com o grau de interesse e de necessidade em relação ao tema, a análise de conteúdo foi utilizada para processar dados, o que foi auxiliado pelo software Atlas.ti 7, com o qual foi estabelecida uma via de reflexão crítica que possibilitou diagnosticar o progresso do conhecimento sobre as questões socioambientais e sua articulação com a formação de professores de ciências. Como resultado, foram estabelecidas quatro categorias nodais emergentes, que refletem a situação atual do campo ambiental na educação em ciências, como um elemento contextualizador cultural, político, ético e crítico, a fim de enfrentar diferentes situações de crise socioambiental.

Palavras-chave: questões socioambientais, educação científica, educação ambiental, formação de professores, estado da arte 
El estado del arte es una modalidad de la investigación documental que permite el estudio del conocimiento acumulado escrito dentro de un área específica. Su finalidad es dar cuenta del sentido del material documental sometido a análisis, con el fin de revisar de manera detallada y cuidadosa los documentos que tratan sobre un tema específico. Es una recopilación crítica de diversos tipos de texto de un área o disciplina, que formaliza el proceso cognitivo de una investigación a través de la lectura de la bibliografía hallada durante la indagación del problema, de los temas y de los contextos (Londoño et al., 2014).

Además, se caracteriza por dos dimensiones:

- La reflexión filosófica sobre la estructura y condiciones del 'comprender'.

- La teoría-práctica de un método que incluye orientaciones para comprender e interpretar la realidad.

Para el desarrollo del presente estado del arte, se tomaron constructos de la concepción filosófica hermenéutica desde un fundamento abductivo. La hermenéutica - del griego hermeneutiké tekhné- es la capacidad para explicar, traducir, interpretar y explicar las relaciones existentes entre un hecho y el contexto en el que acontece. La abducción se refiere al proceso de estudiar los hechos e idear un marco teórico para explicarlos. Se ubica en un espacio intermedio entre la deducción y la inducción, donde se generan hipótesis en el caso de la ausencia de un claro conocimiento teórico inicial, conceptos precisos o teorías elaboradas, situación común cuando se traten cuestiones escasamente investigadas o simplemente novedosas (Verd \& Lozares, 2016).

El fin de esta investigación documental radica en establecer una ruta de reflexión crítica, que permita diagnosticar el avance del conocimiento en el abordaje de cuestiones socioambientales [CSA] y la manera como se articulan con las diversas propuestas de formación del profesorado de ciencias, para este caso como articulador curricular en la Licenciatura en Ciencias Naturales y Educación Ambiental de la Corporación Universitaria Minuto de Dios.

\section{Metodología}

La metodología consistió en la lectura, análisis, interpretación, clasificación y correlación de la información, según el grado de interés y de necesidad frente a la investigación. También se empleó el análisis de contenido para el tratamiento de los datos, el cual fue asistido por el software ATLAS.ti 7. Este procedimiento se sintetiza en la tabla 1.

Tabla 1

Procedimiento metodológico

\begin{tabular}{ccccc}
\hline Concepción & Metodología & Fundamento & Análisis de datos & Instrumento \\
\hline Hermenéutica & Estado del arte & Abductivo & Análisis de contenido & Asistido por ATLAS.ti \\
\hline
\end{tabular}

Según la lógica abductiva -categorías teóricas/categorías emergentes, deducción/ inducción-, inicialmente se definieron categorías a priori del tema de investigación, que permitieron la definición de los descriptores del tesauro. De acuerdo con esto, los descriptores de consulta fueron: ambientalización de la educación superior, formación de profesores, sustentabilidad ambiental, conocimiento didáctico del contenido ambiental, 
educación ambiental crítica, conflictos socioambientales y justicia ambiental.

Una vez definidos los descriptores, se hizo el muestreo documental. Para esta fase se consultaron diversas bases de datos internacionales como Scopus, ERIC, TESEO, EArte, Google Scholar, ProQuest, Dialnet, Redalyc y Web of Science, según la metodología planteada por Mora (2018), descrita en la figura 1.

\section{Figura 1}

Ruta para la construcción del estado del arte

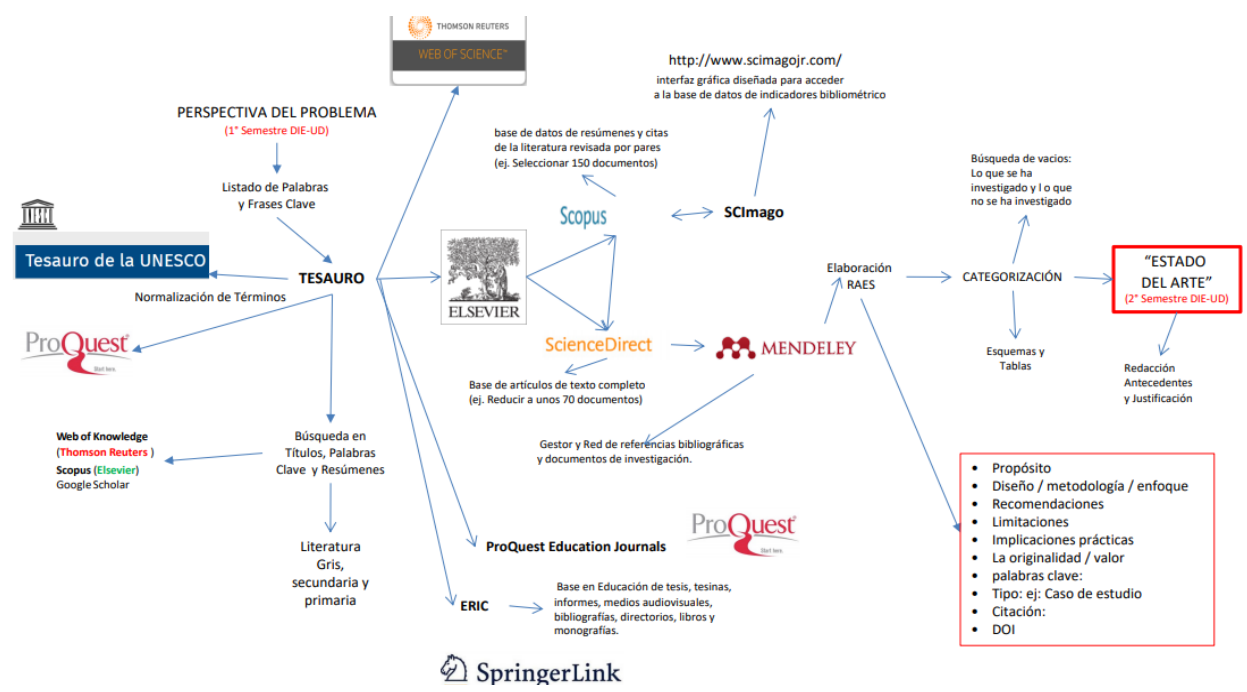

Nota: Tomado de "La metodología de investigación en tesis doctorales: el caso de la línea 'inclusión de la dimensión ambiental en la educación en ciencias"' de W. Mora, 2018, Research strategy, culture development and doctoral support: tools and techniques for latin american universities.

En la información consultada se encontraron handbooks, revistas especializadas, libros, memorias de conferencias, congresos, seminarios, entre otros. Las fuentes documentales encontradas se pueden organizar, según Mora (2018), en tres grandes campos: literatura primaria - textos originales, informes de investigación, artículos de revistas, monografías, bases de datos-, literatura secundaria —resúmenes o referencias de la literatura primaria, catálogos, handbooks, enciclopedias, índices de impacto, resúmenes de disertaciones- y literatura gris - comunicaciones, videos, actas, normas, archivos multimedia-.

En seguida, se realizó un primer análisis de los documentos recopilados. Se revisaron título, resumen, autor, palabras clave y, en algunos documentos, las conclusiones. Con este nuevo filtro se organizaron 210 documentos en una matriz en la que se registraron los vacíos, tendencias y proyecciones investigativas en el tema consultado. Una vez elaborada la matriz bibliográfica de descriptores y resúmenes analíticos, se procedió a realizar una segunda matriz analítica de contenido, en la cual se procuró que los documentos por incluir en esta segunda matriz fueran los que mayor relación guardaran con el objeto de la investigación. Luego de revisar la matriz analítica de contenido y la matriz de antecedentes, se recopilaron 70 documentos entre literatura primaria, secundaria y gris.

Luego de recopilar los documentos y ubicarlos en los campos de la matriz, se requirió identificar unas nuevas categorías denominadas categorías emergentes. Para determinar estas nuevas categorías es necesario revisar detalladamente cada uno de los documentos y 
emplear una lectura minuciosa para interpretar la información con el apoyo de la definición de dichas categorías. Para esta fase, se empleó el software ATLAS.ti 7, una herramienta para realizar análisis cualitativo de información, análisis de contenido y generación de teoría fundamentada en los datos, en coherencia con la metodología propuesta para el desarrollo del presente estado de arte.

\section{Resultados y discusión}

El análisis de contenido requirió de dos procedimientos: el primero fue la definición de las familias de los documentos primarios; y el segundo, la definición de las categorías emergentes y el procedimiento del análisis de contenido como tal. En el primer proceso, se asignaron los documentos recopilados de acuerdo con las categorías a priori con las que fueron inicialmente identificados. Con este procedimiento se generaron cuatro familias de documentos primarios.

La figura 2 muestra la familia alternativas de ambientalización en la educación y los documentos primarios asociados. En el gráfico es posible identificar el nombre del artículo, libro, conferencia, entre otros, y su respectiva codificación al interior del software en la unidad hermenéutica creada.

\section{Figura 2}

Familia de documentos primarios: alternativas de ambientalización en la educación

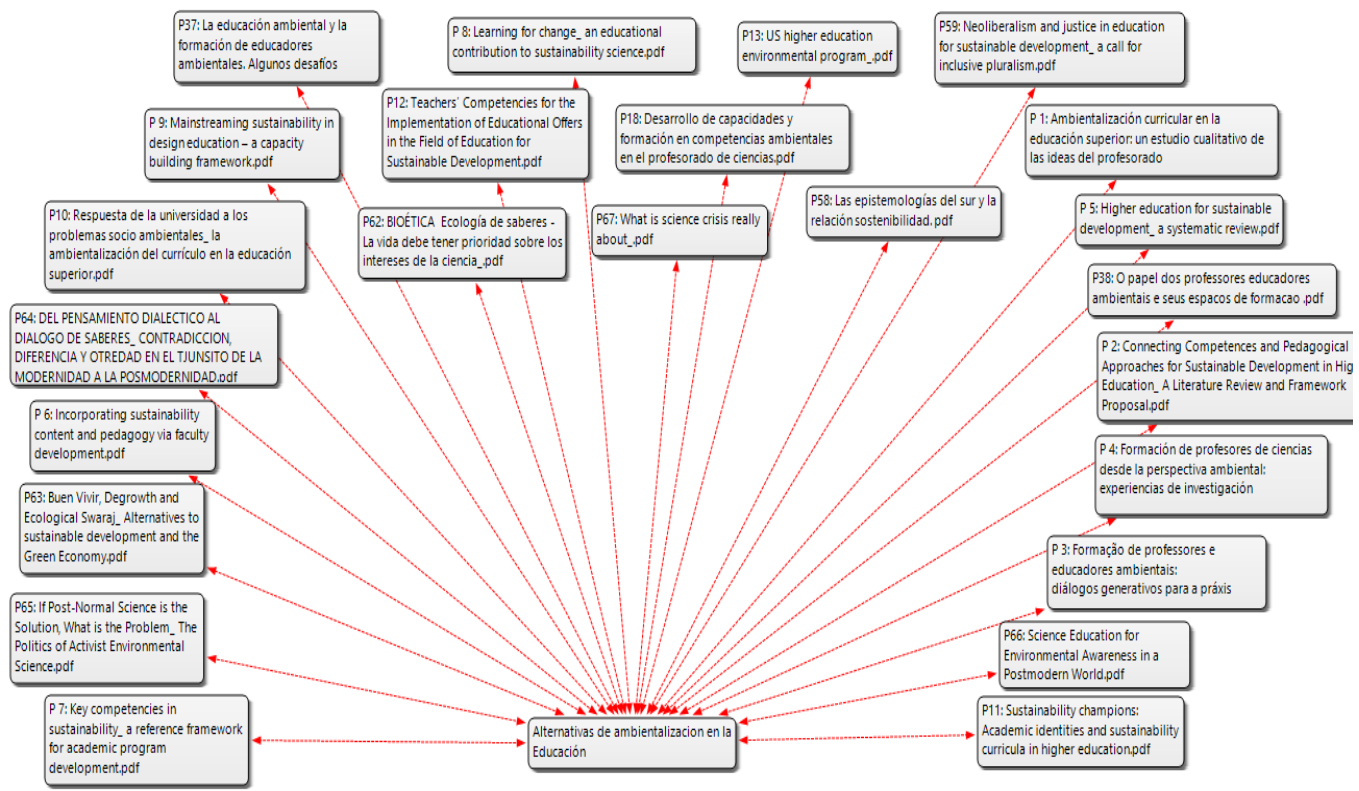

Con este mismo procedimiento, la figura 3 muestra la familia recursos y diseño curricular; la figura 4, la familia resignificación ambiental y educación ambiental crítica; y la figura 5, la familia cuestiones, retos y conflictos socioambientales; todas con sus documentos primarios asociados.

Para el segundo proceso, se leyeron estos 70 documentos y se señalaron citas sobre los textos. A cada cita se le asignó un código abierto. De acuerdo con Hernández et al. (2014), dicho código tiene como función generar una primera categoría, que es definida como emergente en vista de que se obtiene dentro de la lectura de cada documento. Para el 
desarrollo de la investigación, estos códigos serán entendidos como categorías emergentes.

Se identificaron 43 categorías emergentes, por lo cual fue necesario agruparlas. Como criterio para esta agrupación, se tuvieron en cuenta las relaciones entre las categorías emergentes encontradas y el enfoque que le dieron los autores al documento. Este primer agrupamiento es considerado una codificación axial, que fue la base de la siguiente reagrupación en el proceso de análisis. Como resultado de la codificación axial, se obtuvieron 13 categorías.

Figura 3

Familia de documentos primarios: recursos y diseño curricular

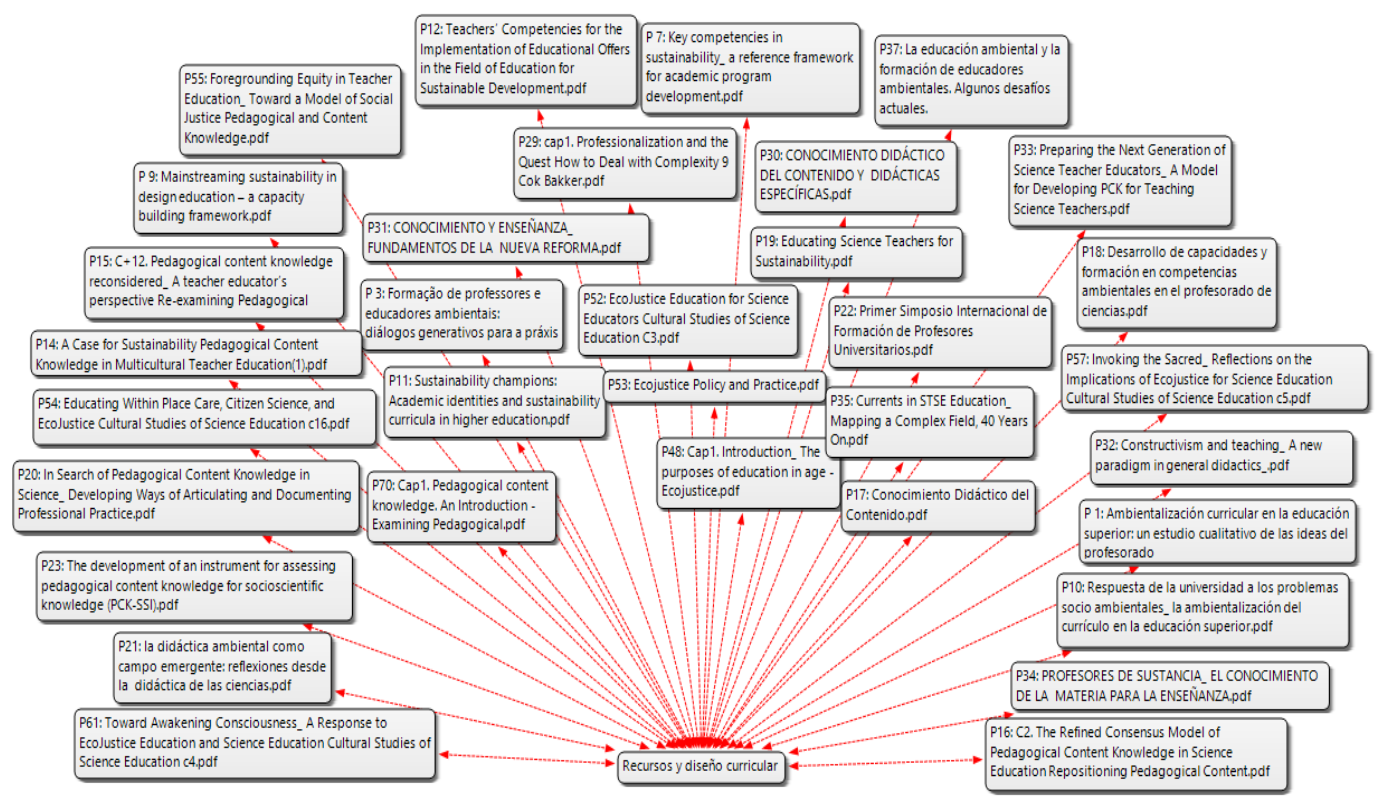

Figura 4

Familia de documentos primarios: resignificación ambiental y educación ambiental crítica

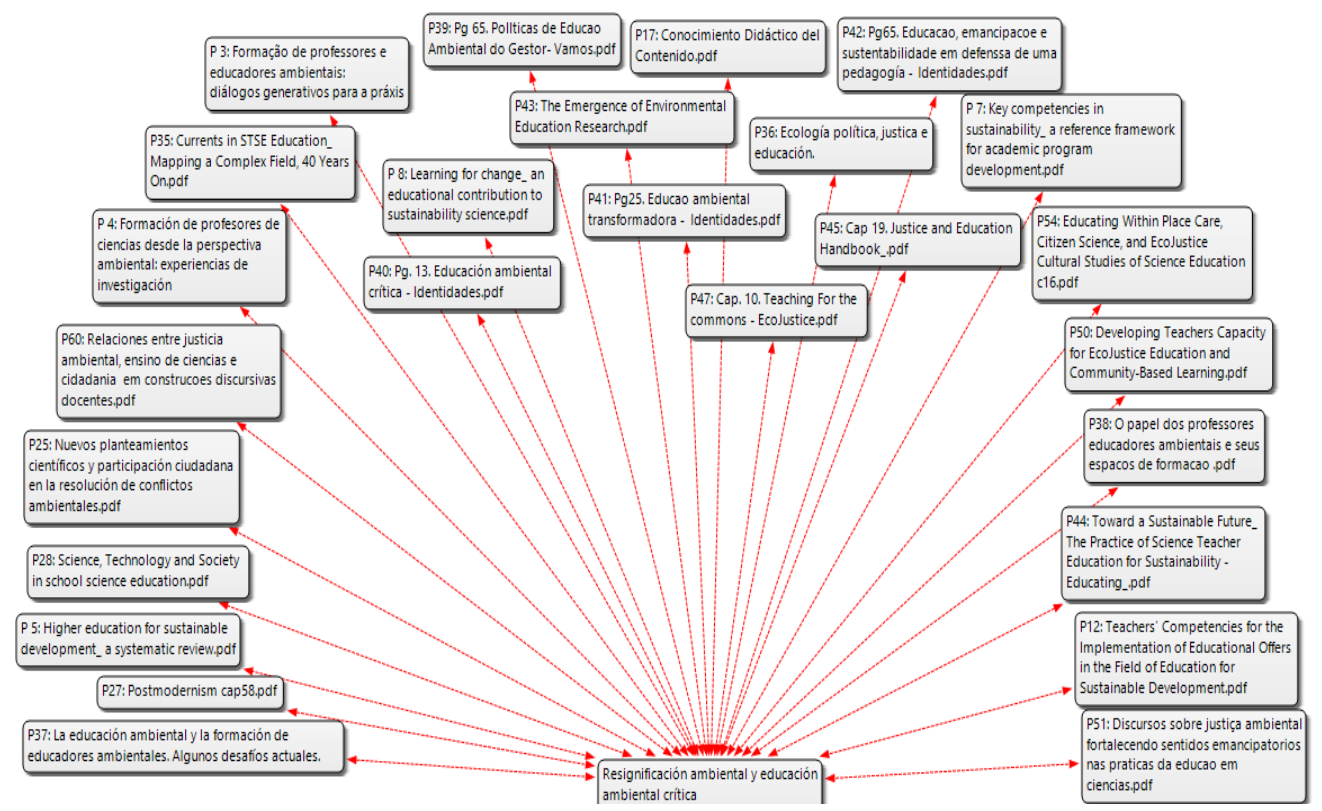


Figura 5

Familia de documentos primarios: cuestiones, retos y conflictos socioambientales

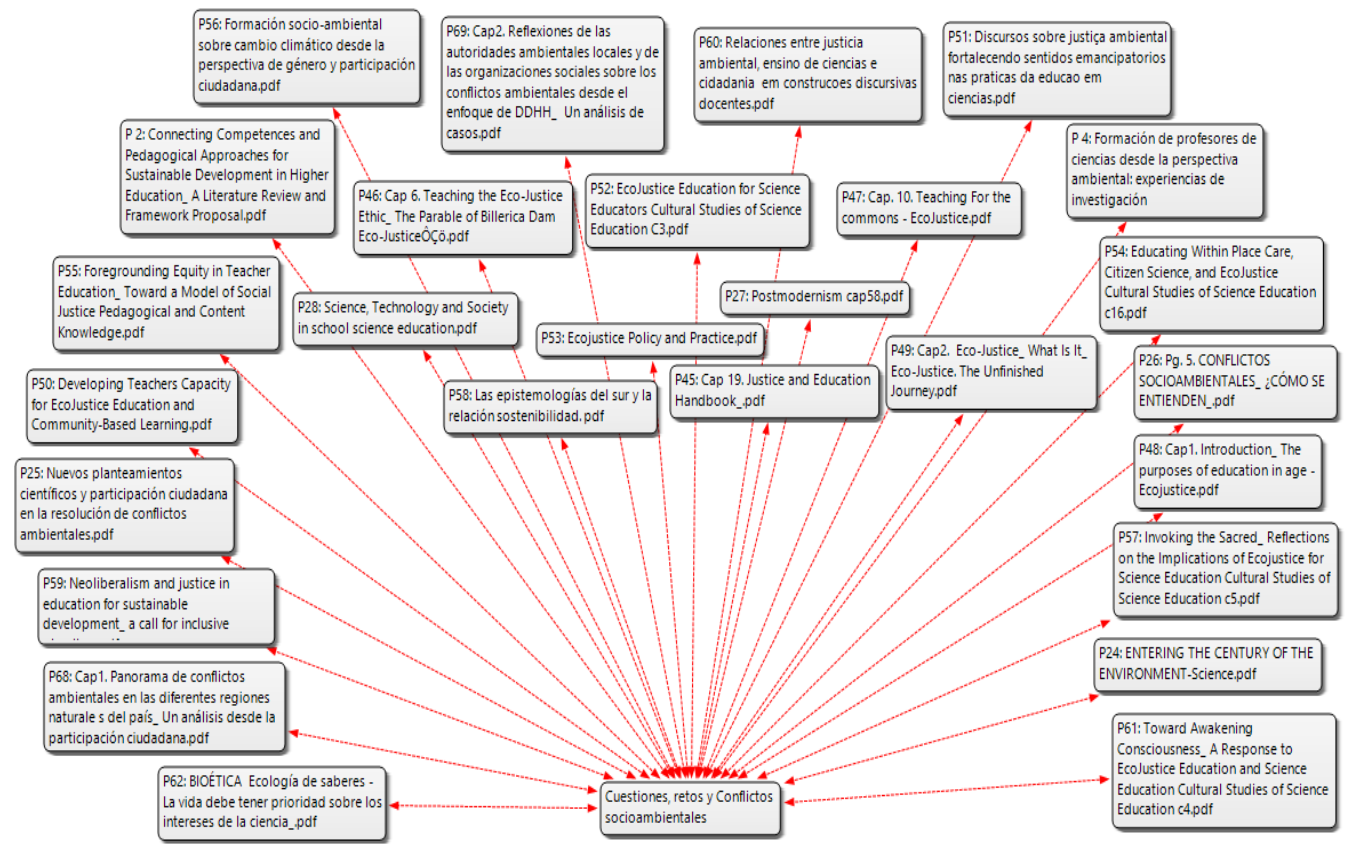

Las categorías emergentes de codificación axial fueron organizadas de acuerdo con el siguiente criterio de agrupación:

- Relación sociedad-naturaleza: las citas que hablan de la relación individuo, naturaleza, territorio. La dimensión crítica de la educación ambiental que busca la emancipación es fundamental para llegar a sociedades sustentables, al mismo tiempo que se evidencia una deficiencia sobre su significado en ese campo de praxis.

- Pensamiento crítico, sistémico, complejo y holístico: las citas que exponen formas de pensamiento holístico, sistémico, complejo y crítico en relación con la relación sociedadnaturaleza.

- Interdisciplinariedad: las citas que le dan un papel relevante a la interdisciplinariedad como elemento transformador.

- Transformación ambiental: las citas que hablan de alternativas para transformar los conflictos ambientales.

- Educación para la sostenibilidad: las citas que hablan del movimiento pedagógico que reconoce la importancia del balance entre el ambiente, la sociedad y la economía.

- Educación para la sustentabilidad: las citas que hablan del movimiento pedagógico que reconoce la importancia de proteger la salud, la cultura, la diversidad y el bienestar de las comunidades locales. Se diferencia de la "sostenibilidad" al desarrollar una comprensión de las tensiones entre culturas y sistemas de racionalidad en las problemáticas de la relación sociedad-naturaleza.

- Ecojusticia: las citas que exponen el estudio de las desigualdades sociales generadas por la distribución inequitativa de los beneficios derivados del uso de la naturaleza y cómo se pueden llevar a cabo políticas orientadas a favorecer una distribución más 
justa. También se exponen los principios de la educación en ecojusticia, entendida como un movimiento pedagógico de responsabilidad y compromiso moral, en defensa del derecho a la permanencia en un espacio natural sentido como propio, protegido del crecimiento y de la inversión incontrolados, de la contaminación, del acaparamiento de tierras, de la especulación, de la desinversión, de la decadencia y del abandono, con el fin de sostener el patrimonio cultural y ambiental del planeta.

- Competencias para la sostenibilidad: las citas que involucran las competencias que se deben tener para el desarrollo sostenible por parte de los profesores y de la sociedad.

- Pedagogía: las citas que hablan de las teorías y posturas pedagógicas y analizan el constructivismo dentro de un entorno social y cultural. Se agrega una dimensión crítica dirigida a transformar estos entornos para mejorar el éxito del constructivismo aplicado como referente.

- Enseñanza: las citas que hablan de los procedimientos para la planificación de las actividades académicas en distintos niveles, el diseño macrocurricular, mesocurricular y microcurricular.

- Identidad del educador en ciencias: las citas que hablan de los roles del profesor de ciencias, la formación en la praxis, la relación profesor-estudiante y la formación de profesores de ciencias y de educadores ambientales.

- Barrera educación ambiental: las citas que involucran barreras externas, de contexto, políticas, barreras individuales, en competencias y roles para la educación ambiental.

- Modelos de desarrollo económico-modernidad y postmodernidad: las citas que exponen los modelos económicos y sus impactos en términos socioambientales, así como la influencia de las corrientes de pensamiento modernidad y posmodernidad.

En la figura 6 se pueden observar las categorías emergentes — códigos y supercódigos-. Cada una de estas categorías está fundamentada. Enseñanza es la mayor fuente de fundamentación, con 154 citas. Le siguen identidad del educador en ciencias con 102, educación para la sostenibilidad con 101, pedagogía con 82 y competencias para la sostenibilidad con 76 . Se entiende como fundamentación al número de citas o referencias que han sido señaladas. Estas se encuentran dentro de los 70 documentos leídos. Luego de haber definido las categorías, fue necesario fijar la densidad entre estas. La densidad se refiere a las relaciones entre una u otra categoría. Los valores se pueden observar en la columna densidad frente a la categoría emergente.

Con base en las ideas de los autores, en las citas y en las categorías asignadas en el proceso de saturación, se establecieron las diferentes relaciones de cohesión, denominadas densidad.

La figura 7 muestra la densidad de las categorías vistas en conjunto. Las relaciones son identificadas por flechas que salen de una categoría y se conectan con otra. Algunas de estas relaciones se muestran como "está asociada con" otra categoría. También hay otras que muestran ser "causa de" otra categoría o que una categoría "es parte" de otra. Sin embargo, se pudo evidenciar una relación de "contradicción" en las categorías emergentes educación para la sustentabilidad y educación para la sostenibilidad. Además, los colores asociados a las categorías diferencian el número de densidades de una categoría a otra. 
Figura 6

Listado de códigos emergentes y supercódigos

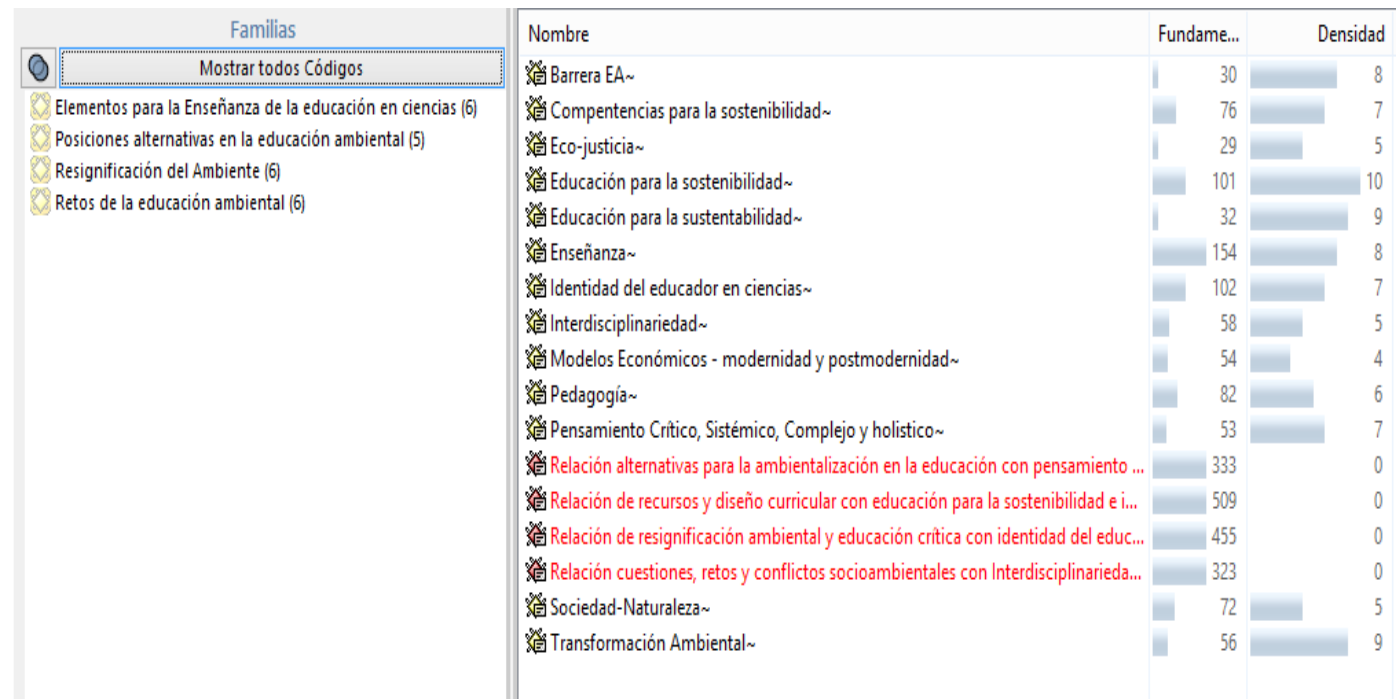

\section{Figura 7}

Relaciones entre códigos o categorías emergentes

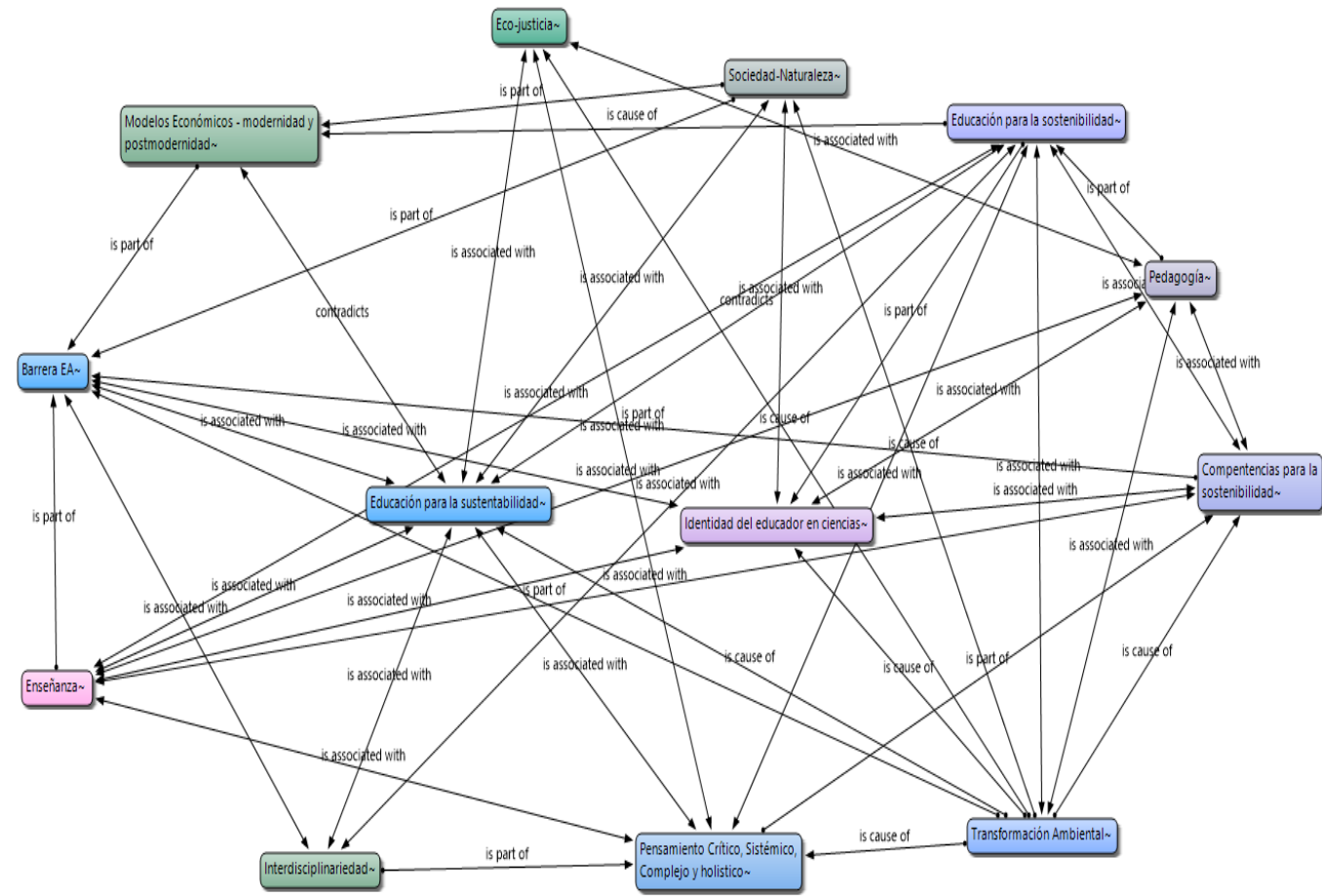

Para continuar con el proceso, se agruparon nuevamente las categorías emergentes, lo cual se conoce como codificación nodal. Allí se deben obtener las familias de códigos empleadas en esta investigación. Para realizar dicha codificación, fue necesario apoyarse en unos supercódigos o supercategorías. Así fue posible tener una mirada holística de las categorías ya establecidas para reagruparlas con el uso de las familias de códigos.

En la figura 8 se puede observar que, en la medida en que se avanza en la codificación, el número de categorías disminuye. Por lo tanto, las categorías que se ubican en la codificación nodal poseen mayores interrelaciones que las anteriores. Es decir, abarcan un mayor número 
de categorías entre sí.

\section{Figura 8}

Secuencia de codificación realizada en Atlas.ti

\section{Documentos primarios}

70

\section{Codificación \\ axial}

13

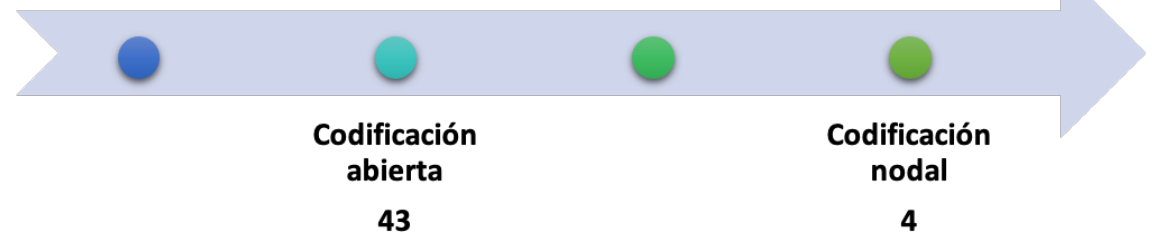

Estas categorías surgen del análisis de las diferentes relaciones en la codificación axial y la codificación abierta. En consecuencia, las categorías que emergen de esta codificación nodal son 4:

- Resignificación del ambiente: este supercódigo relaciona la familia de códigos emergentes resignificación ambiental con rol del educador ambiental, metodología y didáctica (figuras 9 y 10).

- Posiciones alternativas en la educación ambiental: relaciona la familia de códigos emergentes alternativas para la educación ambiental y dicotomías con pensamiento sistémico, complejo y holístico, y didáctica (figuras 11 y 12).

- Elementos para la enseñanza de la educación en ciencias: relaciona la familia de códigos emergentes recursos para la educación ambiental con educación para la sostenibilidad e interdisciplinariedad (figuras 13 y 14).

- Retos de la educación ambiental: relaciona la familia de códigos emergentes retos y barreras ambientales con interdisciplinariedad, ecología política, formación docente y relación individuo-sociedad-ambiente (figuras 15 y 16).

\section{Figura 9}

Relaciones de la categoría nodal "resignificación del ambiente" con las categorías de la codificación axial

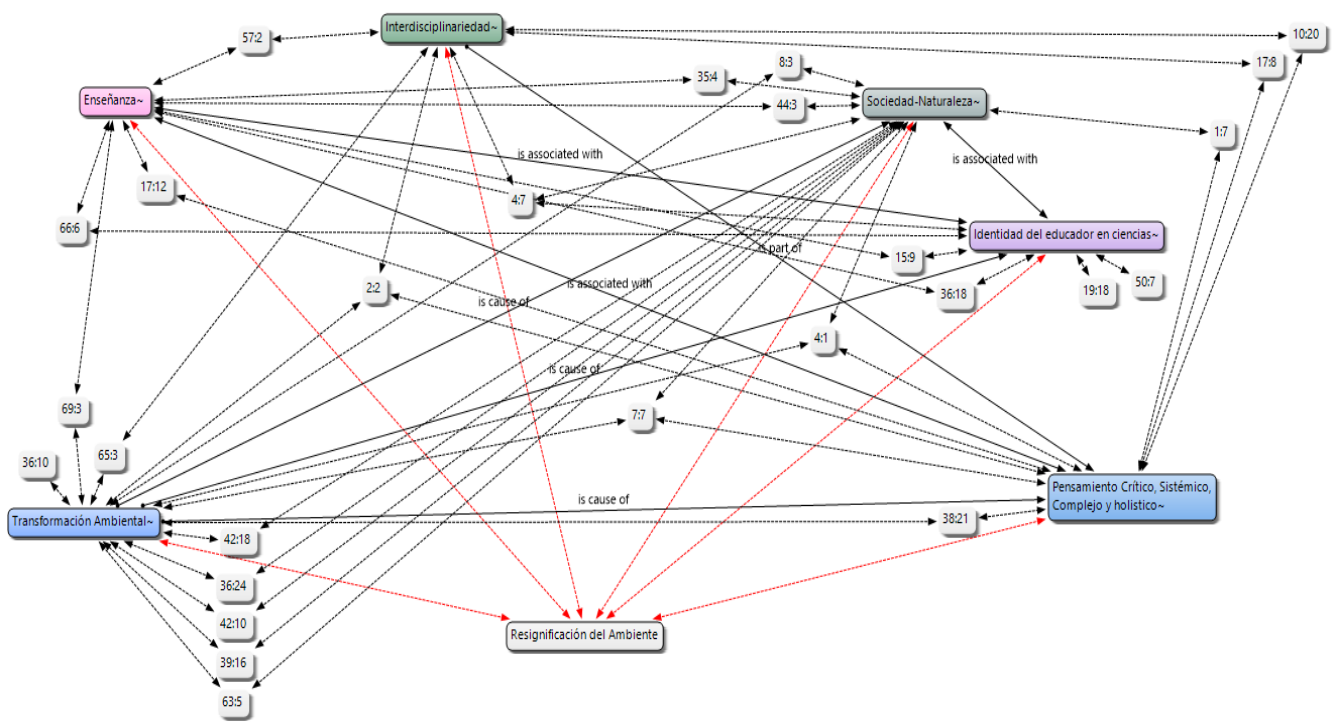




\section{Figura 10}

Detalle de la cita 4:1 en la categoría nodal "resignificación del ambiente"

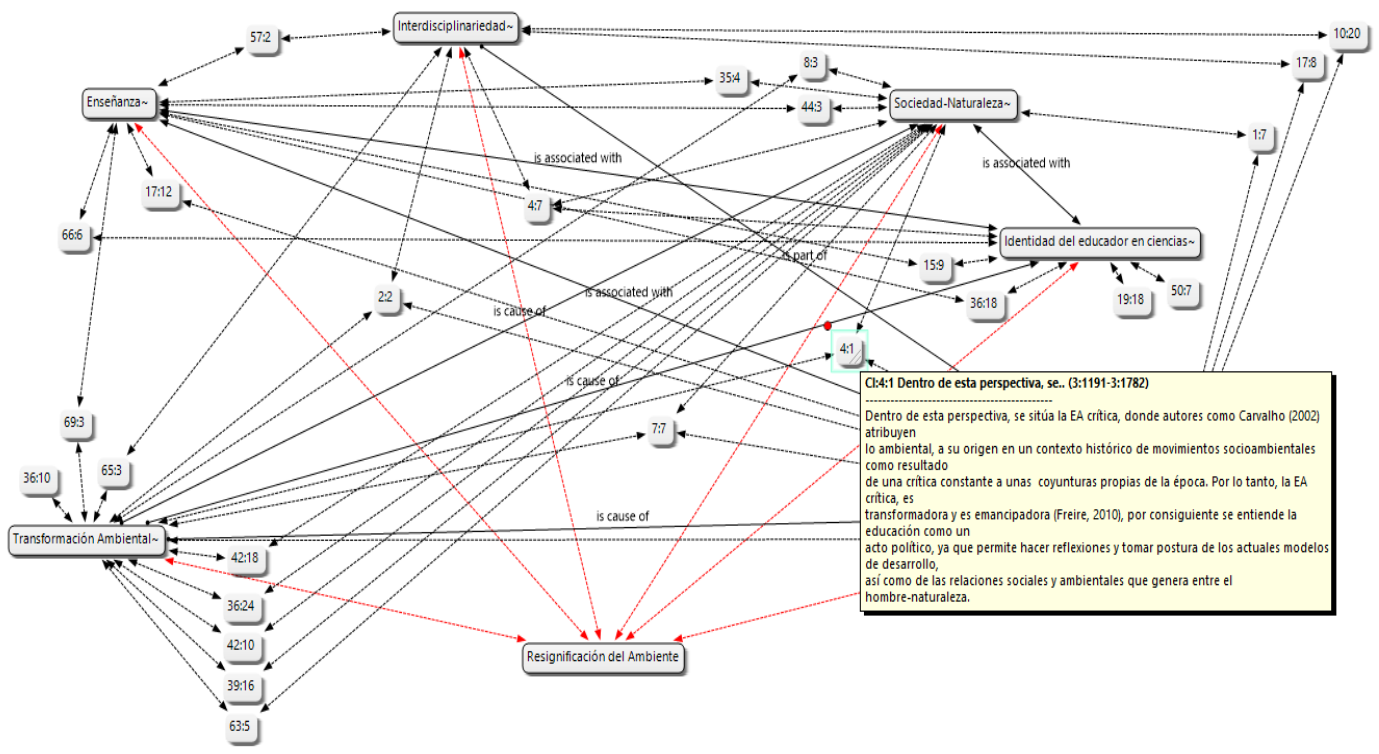

\section{Figura 11}

Relaciones de la categoría nodal "posiciones alternativas en la educación ambiental" con las categorías de la codificación axial

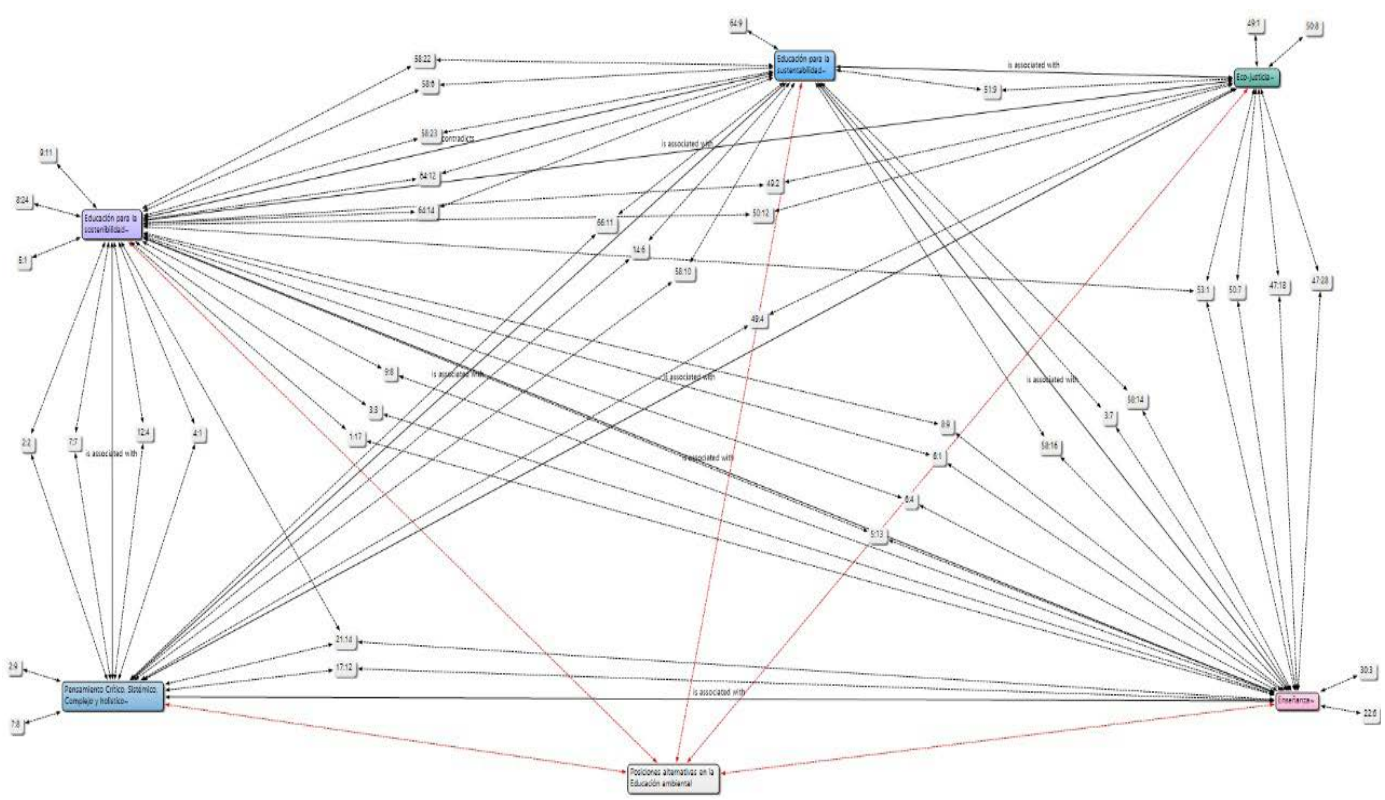




\section{Figura 12}

Detalle de la cita 64:14 en la categoría nodal "posiciones alternativas en la educación ambiental"

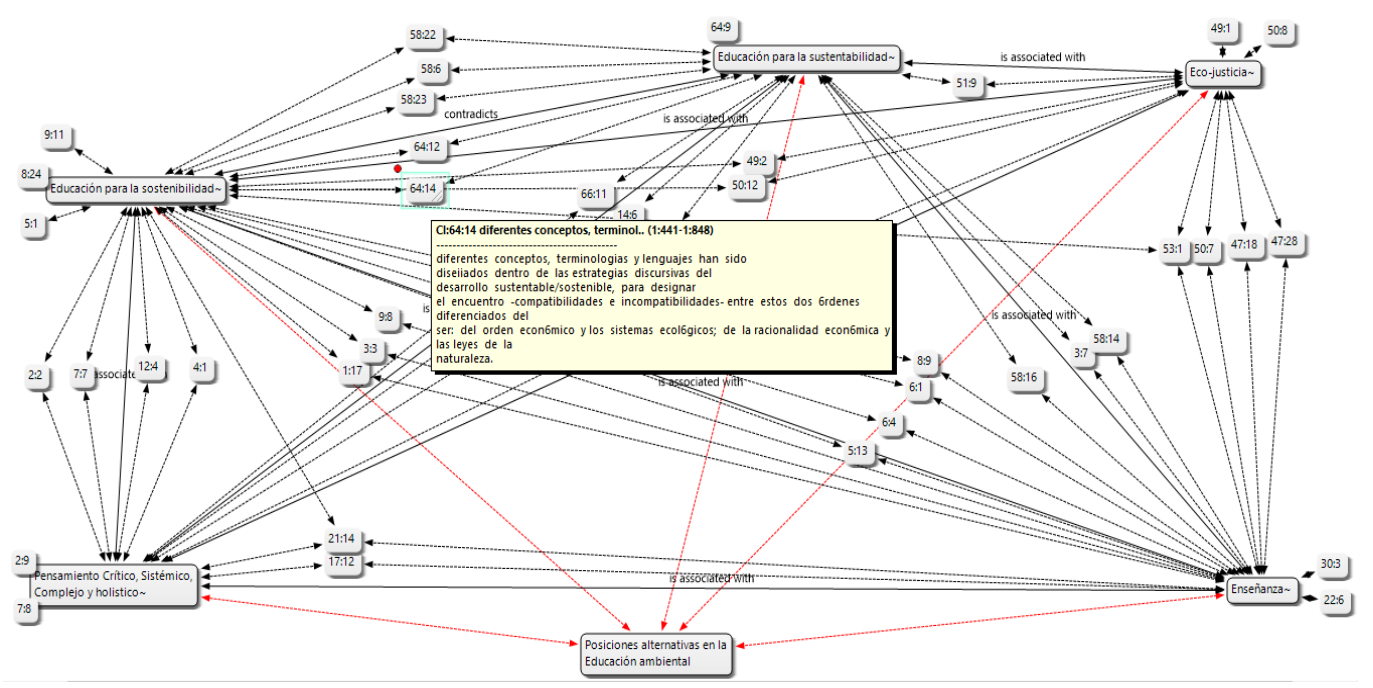

\section{Figura 13}

Relaciones de la categoría nodal "elementos para la enseñanza de la educación en ciencias" con las categorías de la codificación axial

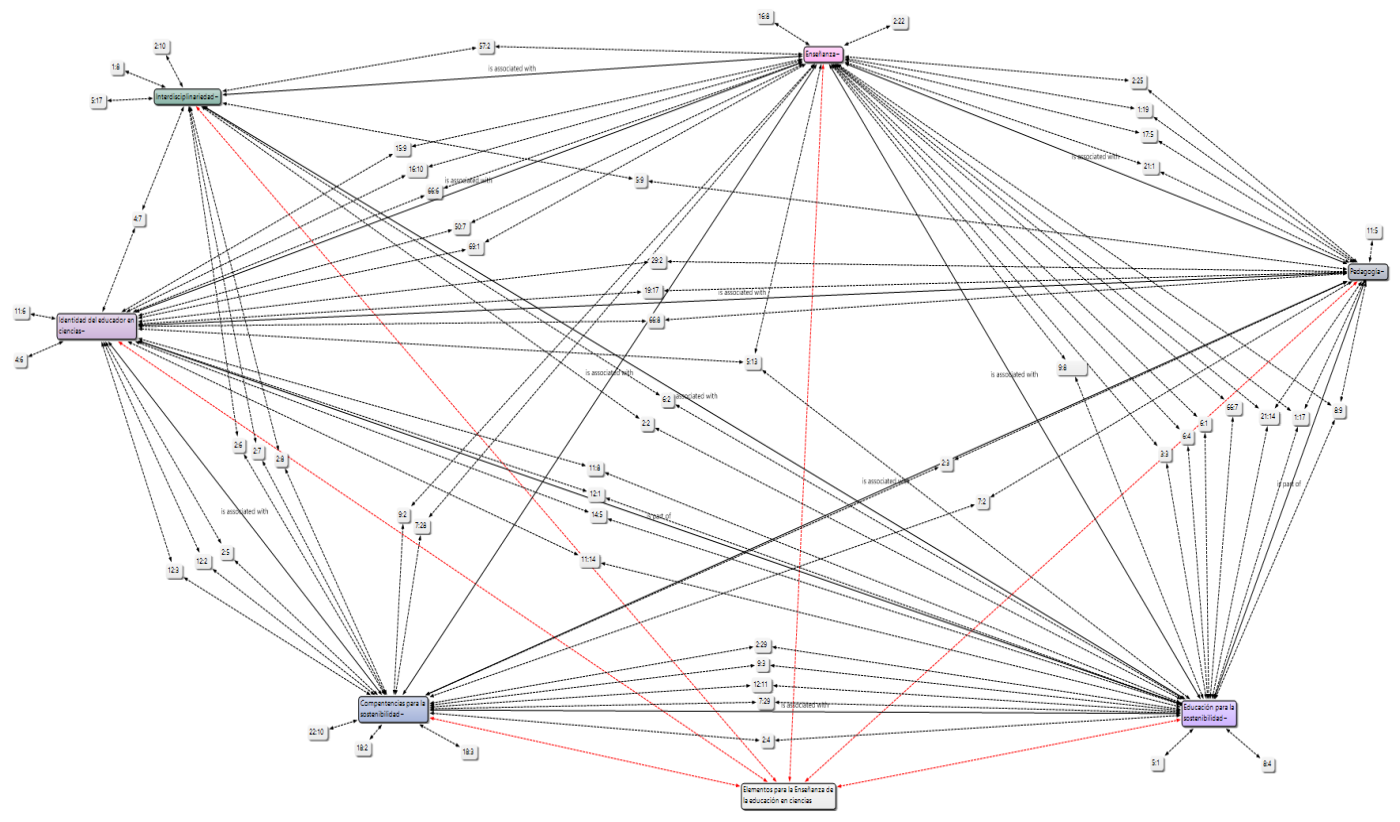




\section{Figura 14}

Detalle de la cita 5:13 en la categoría nodal "elementos para la enseñanza de la educación en ciencias"

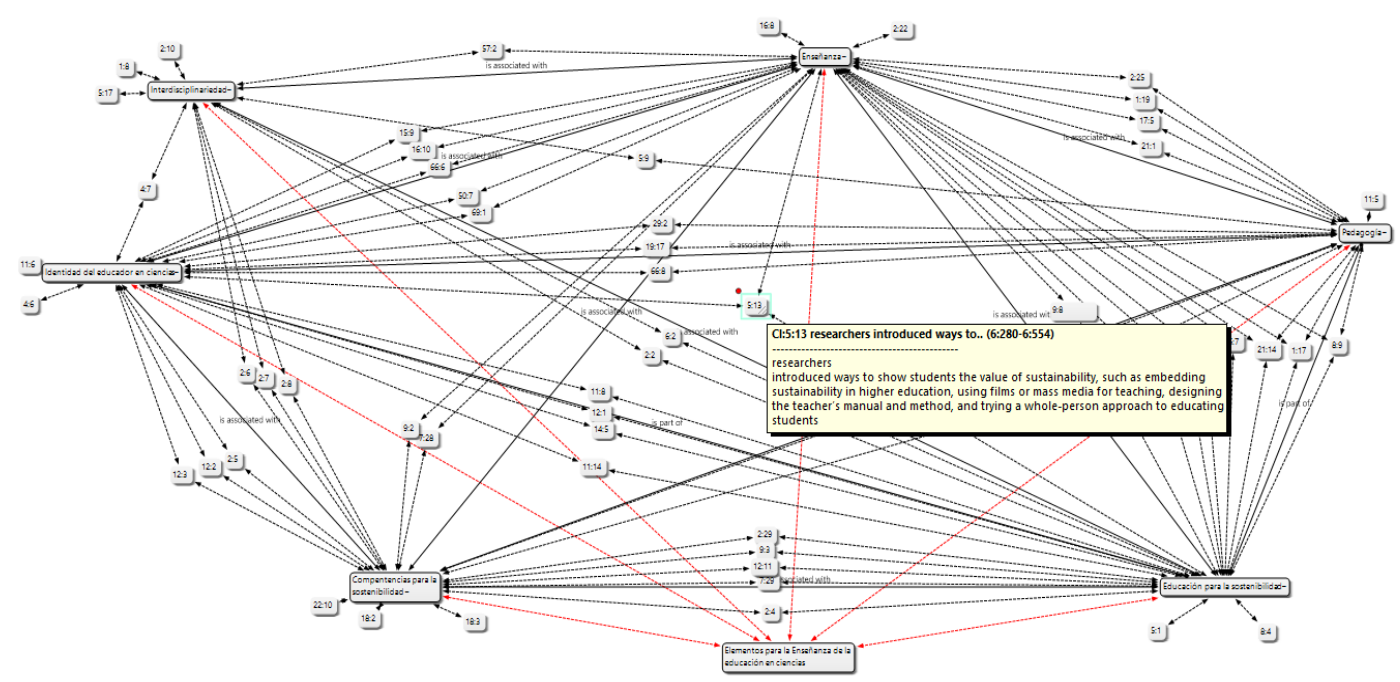

\section{Figura 15}

Relaciones de la categoría nodal "retos de la educación ambiental" con las categorías de la codificación axial

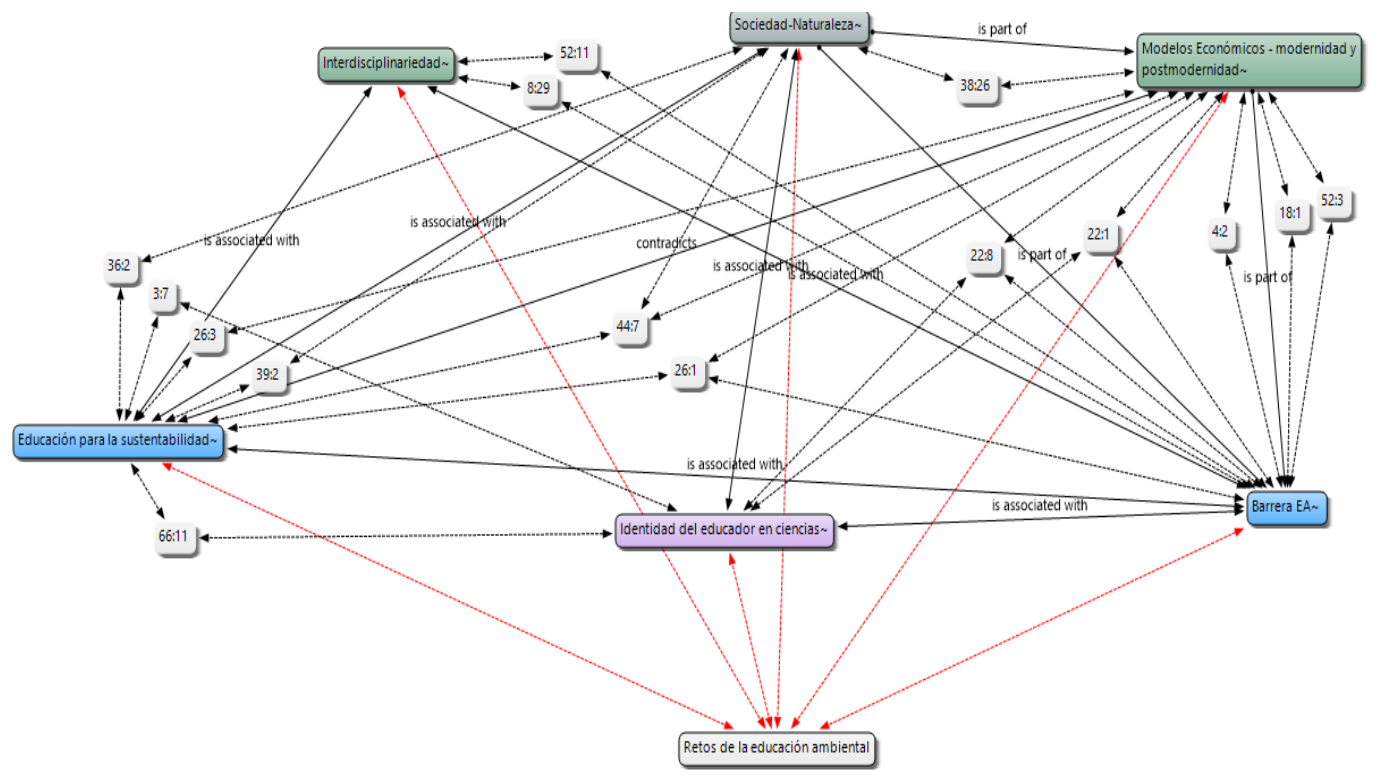




\section{Figura 16}

Detalle de la cita 22:8 en la categoría nodal "retos de la educación ambiental"

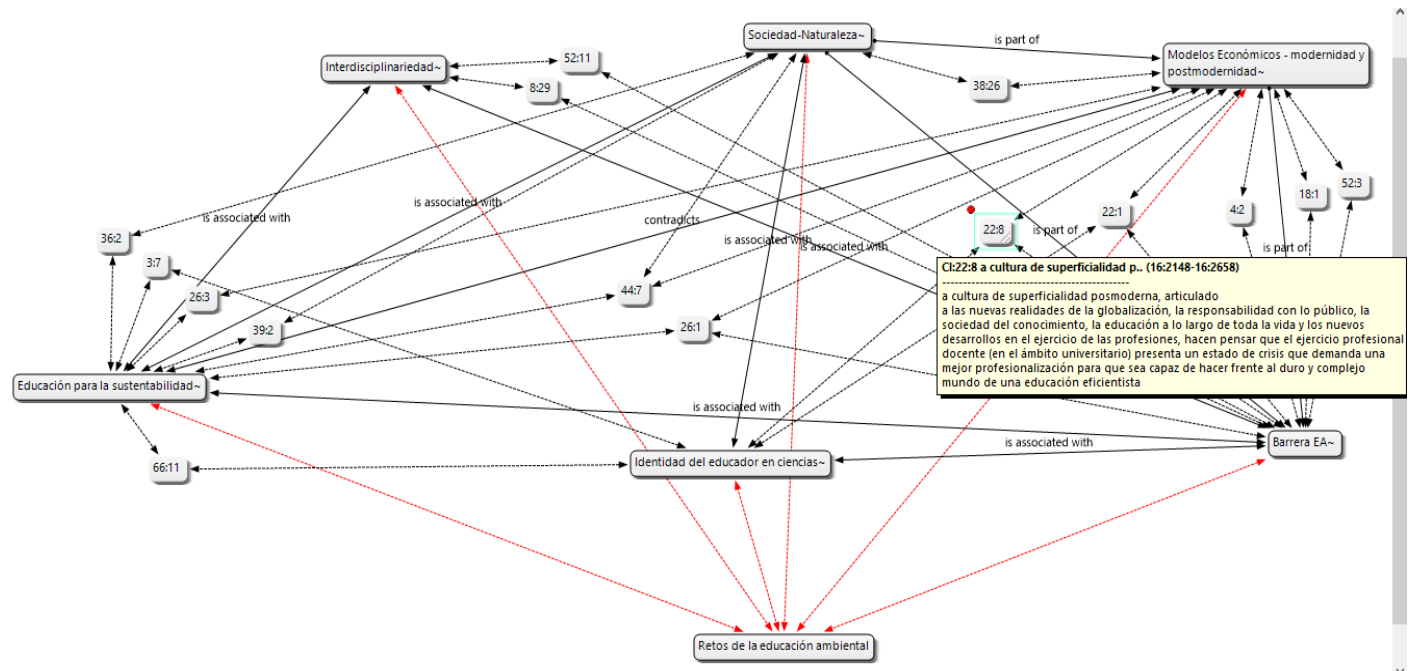

Para establecer las cuatro categorías nodales — núcleo- emergentes - resignificación del ambiente, posiciones alternativas en la educación ambiental, elementos para la enseñanza de la educación en ciencias y retos de la educación ambiental-, se partió de la teoría fundamentada - grounded theory-. Es decir, la teoría entendida como los hallazgos fue emergiendo fundamentada en los datos. Como lo afirma Creswell (2013), se trata de un proceso no lineal, que surge a partir de los datos.

En este sentido, se puede evidenciar en las figuras 9 a 16 cómo se amplía la información relevante sobre las múltiples relaciones que existen entre las categorías nodales con algunas categorías emergentes del proceso de codificación axial. Los números anidados entre categorías asocian una cita perteneciente a alguno de los 70 documentos primarios con los cuales se inició el proceso de investigación documental. Estas citas pueden estar asociadas a dos o más categorías. El primer número se refiere al documento y el segundo a la cita generada. Es importante mencionar que en el proceso de saturación se ha demostrado que existe un gran número de citas asociado a cada código.

La presente aproximación a un estado del arte explora la interrelación entre la educación en ciencias y la educación ambiental, debido no solo a las limitantes de cada una de las dos educaciones, sino a la pragmática de los profesores de ciencias frente al tema ambiental.

Los hallazgos dan cuenta de las relaciones que se suscitan entre las CSA y la educación en justicia ambiental como elementos articuladores en la educación en ciencias, al tomar referentes conceptuales y teóricos de la educación en ecojusticia y la educación ambiental crítica, con el fin de promover una enseñanza para la participación ciudadana y la formación en valores y actitudes para la acción, en el marco de la formación de profesores de ciencias naturales.

En la actualidad se evidencia la urgencia de incluir la dimensión ambiental en el currículo de formación y en el quehacer del profesorado de ciencias, ya que los conflictos socioambientales - propios de la relación sociedad-naturaleza- persisten. Es responsabilidad y tarea de la comunidad educativa dar respuesta en la búsqueda de alternativas a los retos que impone la creciente problemática ambiental. 
Finalmente, con base en estas cuatro categorías nodales, se presenta la siguiente matriz analítica que contiene los documentos trabajados (tabla 2).

Tabla 2

Matriz analítica

\begin{tabular}{|c|c|c|c|c|}
\hline Documento & $\begin{array}{c}\text { C.E. } \\
1\end{array}$ & $\begin{array}{l}\text { C.E. } \\
2\end{array}$ & $\begin{array}{c}\text { C.E. } \\
3\end{array}$ & $\begin{array}{c}\text { C.E. } \\
4\end{array}$ \\
\hline Acselrad \& Mello (2009). O que é justiça ambiental. & & $\mathrm{X}$ & & \\
\hline Alier (2007). O ecologismo dos pobres. & $\mathrm{X}$ & & & \\
\hline $\begin{array}{l}\text { Bentley (2010). Toward awakening consciousness: a response to ecojustice } \\
\text { education and science education. }\end{array}$ & & & $\mathrm{X}$ & \\
\hline Bullard (2004). Enfrentando o racismo ambiental no século XXI . & & & & $\mathrm{X}$ \\
\hline $\begin{array}{l}\text { Cosenza \& Martins (2012). Os sentidos de conflito ambiental na educação } \\
\text { ambiental: uma análise dos periódicos de educação ambiental. }\end{array}$ & & $\mathrm{X}$ & & \\
\hline $\begin{array}{l}\text { Cosenza et al. (2014). Relações entre justiça ambiental, ensino de ciências } \\
\text { e cidadania em construções discursivas docentes. }\end{array}$ & & & $\mathrm{X}$ & \\
\hline $\begin{array}{l}\text { Dyches \& Boyd (2017). Foregrounding equity in teacher education: toward } \\
\text { a model of social justice pedagogical and content knowledge. }\end{array}$ & & & $\mathrm{X}$ & \\
\hline
\end{tabular}

a model of social justice pedagogical and content knowledge.

Flórez \& Prieto (2017). Conflictos socioambientales en los páramos de la $\quad \mathrm{X}$ sabana de Bogotá. Estudios nacionales.

Folchi (2001). Conflictos de contenido ambiental y ecologismo de los po- $\quad \mathrm{X}$ bres: no siempre pobres, ni siempre ecologistas.

Freire, Cáceres \& Freitas (2016). Formación de profesores de ciencias desde la perspectiva ambiental: experiencias de investigación.

Freire, Figueiredo \& Guimarães (2016). O papel dos professores/educadores ambientais e seus espaços de formação. Qual é a educação ambiental que nos emancipa?

Freire \& Rodrigues (2020). Formação de professores e educadores ambientais: diálogos generativos para a práxis.

\begin{tabular}{lcc}
\hline Gibson (2012). Eco-justice. & $\mathrm{X}$ \\
\hline Hackman (2005). Five essential components of social justice education. & $\mathrm{X}$ \\
\hline Haluza (2013). Educating for environmental justice. & & $\mathrm{X}$ \\
\hline $\begin{array}{l}\text { Herman et al. (2018). A socioscientific issues approach to environmental } \\
\text { education. }\end{array}$ & $\mathrm{X}$ & \\
\hline $\begin{array}{l}\text { Juliani et al. (2014). Inserção da educação ambiental na formação inicial } \\
\text { docente: levantamento de publicações. }\end{array}$ & $\mathrm{X}$ \\
\hline
\end{tabular}

Londoño et al. (2012). Conflictos ambientales en Colombia. Retos y pers- $\quad \mathrm{X}$
pectivas desde el enfoque de DDHH y la participación ciudadana.

Loureiro \& Layrargues (2013). Ecologia política, justiça e educação ambiental crítica: perspectivas de aliança contra-hegemônica.

$\mathrm{X}$

\begin{tabular}{lc}
\hline $\begin{array}{l}\text { Martusewicz et al. (2015). EcoJustice education. Toward diverse, demo- } \\
\text { cratic, and sustainable communities. }\end{array}$ & $\mathrm{X}$ \\
\hline $\begin{array}{l}\text { Mesa (2018). Una idea de justicia ambiental. Elementos de conceptualiza- } \\
\text { ción y fundamentación. }\end{array}$ & $\mathrm{X}$ \\
\hline $\begin{array}{l}\text { Mora (2012). Ambientalización curricular en la educación superior: un } \\
\text { estudio cualitativo de las ideas del profesorado. }\end{array}$ & $\mathrm{X}$ \\
\hline $\begin{array}{l}\text { Mora (2015). Desarrollo de capacidades y formación en competencias } \\
\text { ambientales en el profesorado de ciencias. }\end{array}$ & $\mathrm{X}$ \\
\hline $\begin{array}{l}\text { Mora (2016). Problemas ambientales, ciencia posnormal y ética ambiental. } \\
\text { Mora (2018). La metodología de investigación en tesis doctorales: el caso } \\
\text { de la línea "inclusión de la dimensión ambiental en la educación en cien- } \\
\text { cias". }\end{array}$ & $\mathrm{X}$ \\
\hline
\end{tabular}


Mora (2019). Cuestiones socioambientales y justicia socioambiental: diseño curricular y formación docente.

Mora (2020). Las epistemologías del sur y la relación sostenibilidad / sus-

tentabilidad en la construcción conceptual de una línea de investigación didáctica sobre justicia socioambiental.

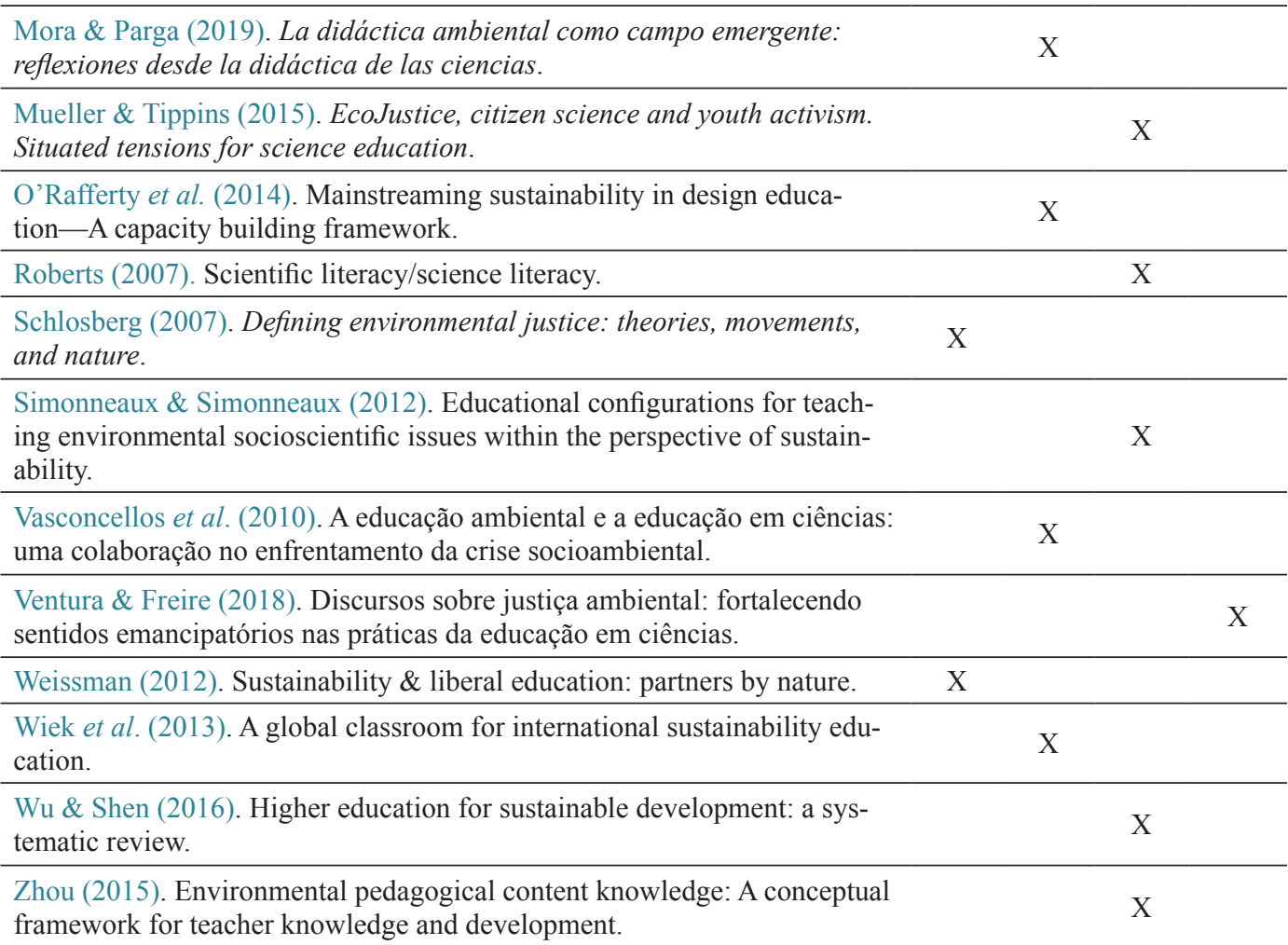

Nota: Adaptada de “The state of the art: a research methodology" de M. Gómez, C. Galeano, \&

D. Jaramillo, 2015, Revista Colombiana de Ciencias Sociales, 6(2). La sigla C. E. hace referencia a categoría emergente.

Para desarrollar y definir los elementos conceptuales que guían la investigación, se tomó como punto de partida la información obtenida de la codificación abierta, axial y nodal del estado del arte. Con las relaciones establecidas, se procedió a tomar las citas más importantes de los documentos primarios, lo que dio cabida a grandes campos conceptuales para definir ocho referentes teóricos: las CSA, la educación basada en CSA, la justicia ambiental, la educación en justicia ambiental, la educación ambiental crítica, la articulación con la educación en ciencias, la formación de profesores de ciencias y la dimensión ambiental en la educación superior.

\section{Conclusión}

Producto de un estudio documental de estado del arte, con el fin de justificar las CSA como articulador curricular de la Licenciatura en Ciencias Naturales y Educación Ambiental de la Corporación Universitaria Minuto de Dios, se han seleccionado 70 documentos, entre literatura primaria, secundaria y gris, procedentes de bases especializadas en educación, que han conducido a establecer cuatro categorías nodales - núcleo-emergentes: resignificación del ambiente, posiciones alternativas en la educación ambiental, elementos para la enseñanza de la educación en ciencias y retos de la educación ambiental, que dan cuenta de la situación actual del campo ambiental situado en lo educativo, particularmente en la educación en 
ciencias, como elemento contextualizador cultural, político, y ético, como ejemplo de una educación ambiental articulada a otras áreas del conocimiento, en pro de enfrentar las diversas situaciones de crisis socioambiental que ponen en situación de incertidumbre a la modernidad.

Se requiere de una educación articuladora entre ciencias y ambiente, dirigida a la participación ciudadana. El desarrollo de capacidades ambientales en los estudiantes está determinado por contextos curriculares propios de conflictos socioambientales reales de justicia socioambiental, que requieren del profesorado una formación pedagógicodidáctica ambientalizada en torno a un conocimiento didáctico del contenido en justicia socioambiental —o social justice pedagogical and content knowledge-.

\section{Referencias}

Acselrad, H., \& Mello, C. (2009). O que é justiça ambiental. Garamond.

Alier, J. (2007). O ecologismo dos pobres. Contexto.

Bentley, M. (2010). Toward Awakening Consciousness: A Response to EcoJustice Education and Science Education. En D. Tippins, M. Mueller, M. van Eijck, \& J. Adams (Eds.), Cultural Studies and Environmentalism (pp. 29-41). Cultural Studies of Science Education, vol 3. Springer, Dordrecht. https://doi.org/10.1007/978-90-481-3929-3_4.

Bullard, R. (2004). Enfrentando o racismo ambiental no século XXI. En H. Acselrad, S. Herculano, \& J. Pádua (Eds.), Justiça ambiental e cidadania (pp. 41-68). Fundação Ford.

Cosenza, A., \& Martins, I. (2012). Os sentidos de conflito ambiental na educação ambiental: uma análise dos periódicos de educação ambiental. Revista Ensino, Saúde, Ambiente, 5(2), 234-245.

Cosenza, A., Freire, L., Espinet, M., \& Martins, I. (2014). Relações entre justiça ambiental, ensino de ciências e cidadania em construções discursivas docentes. Revista Brasileira de Pesquisa em Educação em Ciências, 14(2), 89-98.

Creswell, J. (2013). Educational research: planning, conducting, and evaluating. W. Ross MacDonald School Resource Services Library.

Dyches, J., \& Boyd, A. (2017). Foregrounding equity in teacher education: toward a model of social justice pedagogical and content knowledge. Journal of Teacher Education, 68(5), 476-490.

Flórez, M., \& Prieto, A. (2017). Conflictos socioambientales en los páramos de la sabana de Bogotá. Estudios nacionales. Asociación Ambiente y Sociedad.

Folchi, M. (2001). Conflictos de contenido ambiental y ecologismo de los pobres: no siempre pobres, ni siempre ecologistas. Ecología Política, 22, 79-100. https://dialnet.unirioja.es/ servlet/ articulo?codigo $=153467$.

Freire, L., Cáceres, M., \& Freitas, S. (2016). Formación de profesores de ciencias desde la perspectiva ambiental: experiencias de investigación. Maestría en Docencia de la Química.

Freire, L., Figueiredo, J., \& Guimarães, M. (2016). O papel dos professores/educadores ambientais e seus espaços de formação. Qual é a educação ambiental que nos emancipa? 
Pesquisa em Educação Ambiental, 11(2), 117-125.

Freire, L., \& Rodrigues, C. (2020). Formação de professores e educadores ambientais: diálogos generativos para a práxis. Pesquisa em Educação Ambiental, 15(1).

Gibson, W. E. (Ed.). (2012). Eco-Justice: The Unfinished Journey. SUNY Press.

Gómez, M., Galeano, C., \& Jaramillo, D. (2015). The state of the art: a research methodology. Revista Colombiana de Ciencias Sociales, 6(2), 423-442.

Hackman, H. (2005). Five essential components of social justice education. Equity \& Excellence in Education, 38, 103-109.

Haluza, R. (2013). Educating for environmental justice. En R. Stevenson, M. Brody, J. Dillon, \& A. Wals (Eds.), International Handbook of Research on Environmental Education (pp. 394-403). Routledge.

Herman, B., Sadler, T., Zeidler, D., \& Newton, M. (2018). A socioscientific issues approach to environmental education. En G. Reis, \& J. Scott (Eds.), International Perspectives on the Theory and Practice of Environmental Education: A Reader (pp. 145-161). Springer International Publishing AG. https://doi.org/10.1007/978-3-319-67732-3.

Hernández, R., Fernández, C., \& Baptista, M. (2014). Metodología de la investigación. McGraw-Hill Interamericana.

Juliani, S., Freire, L. M., García, E., \& Rezende Filho, L. A. (2014). Inserção da Educação Ambiental na formação inicial docente: Levantamento de publicações. Revista Tecné, Episteme y Didaxis, (número extraordinario), 1555-1562.

Londoño, B., Güiza, L., \& Muñoz, L. (2012). Confictos ambientales en Colombia. Retos y perspectivas desde el enfoque de DDHH y la participación ciudadana. Editorial Universidad del Rosario.

Londoño, O., Maldonado, L., \& Calderón, L. (2014). Guías para construir estados del arte. IcnK.

Loureiro, C., \& Layrargues, P. (2013). Ecologia política, justiça e educação ambiental crítica: perspectivas de aliança contra-hegemônica. Trabalho, Educação e Saúde, 11(1), 53-71.

Martusewicz, R., Edmundson, J., \& Lupinacci, J. (2015). EcoJustice education. Toward diverse, democratic, and sustainable communities ( $2^{\mathrm{a}}$ ed.). Taylor \& Francis.

Mesa, G. (2018). Una idea de justicia ambiental. Elementos de conceptualización y fundamentación. Universidad Nacional de Colombia.

Mora, W. (2012). Ambientalización curricular en la educación superior: un estudio cualitativo de las ideas del profesorado. Revista de Currículum y Formación de Profesorado, 16(2), 77-103.

Mora, W. (2015). Desarrollo de capacidades y formación en competencias ambientales en el profesorado de ciencias. Revista: Tecné, Episteme y Didaxis, (38), 185-203.

Mora, W. (2016). Problemas ambientales, ciencia posnormal y ética ambiental. En D. Rodríguez. (Ed.), Bioética. Ecología de saberes (pp. 147-162). Universidad Libre. 
Mora, W. (2018). La metodología de investigación en tesis doctorales: el caso de la línea "inclusión de la dimensión ambiental en la educación en ciencias". En D. Edgar, P. Duncan, \& F. Valencia (Eds.), Research strategy, culture development and Doctoral Support: Tools and Techniques for Latin American Universities (pp. 162 - 184). Latin American University Research and Doctoral Support.

Mora, W. (2019). Cuestiones socioambientales y justicia socioambiental: diseño curricular y formación docente. Latin American Journal of Science Education, (6), 22006.

Mora, W. (2020). Las epistemologías del sur y la relación sostenibilidad / sustentabilidad en la construcción conceptual de una línea de investigación didáctica sobre justicia socio-ambiental. En A. Molina (Ed.), Investigación y formación de profesores de ciencias: Diálogos de perspectivas latinoamericanas (pp. 21-54). Universidad Distrital Francisco José de Caldas.

Mora, W., \& Parga D. (2019). La didáctica ambiental como campo emergente: reflexiones desde la didáctica de las ciencias [Ponencia]. Seminario de Educación Ambiental en las Universidades Latinoamericanas: Retos Perspectivas y Apuestas. Universidad del Tolima, Ibagué, Colombia.

Mueller, M. \& Tippins, D. (2015). EcoJustice, citizen science and youth activism. Situated tensions for science education. Springer.

O’Rafferty, S., Curtis, H., \& O’Connor, F. (2014), Mainstreaming sustainability in design education - a capacity building framework. International Journal of Sustainability in Higher Education, 15(2), 169-187. https://doi.org/10.1108/IJSHE-05-2012-0044.

Roberts, D. (2007). Scientific literacy/science literacy. En S. Abell, \& N. Lederman (Eds.), Handbook of research on science education (pp. 729-781). Lawrence Earlbaum.

Schlosberg, D. (2007). Defining environmental justice: theories, movements, and nature. Oxford University Press Inc.

Simonneaux, J., \& Simonneaux, L. (2012). Educational configurations for teaching environmental socioscientific issues within the perspective of sustainability. Research in Science Education, 42(1), 75-94.

Vasconcellos, M., Loureiro, C., \& Queiroz, G. (2010). A educação ambiental e a educação em ciências: uma colaboração no enfrentamento da crise socioambiental. Revista Brasileira de Pesquisa em Educação em Ciências, 10(1), 1-19.

Ventura, G., \& Freire, L. (2018). Discursos sobre justiça ambiental: fortalecendo sentidos emancipatórios nas práticas da educação em ciências. Ensino, Saude e Ambiente Backup, 10(3).

Verd, J., \& Lozares, C. (2016). Introducción a la investigación cualitativa. Fases, métodos y técnicas. Síntesis.

Weissman, N. (2012). Sustainability \& liberal education: partners by nature. Liberal Education, 98(4).

Wiek, A., Bernstein, M., Laubichler, M., Caniglia, G., Minteer, B., \& Lang, D. (2013). A global classroom for international sustainability education. Creative Education, 4(4A), 19-28. 
Wu, Y., \& Shen, J. (2016). Higher education for sustainable development: a systematic review, International Journal of Sustainability in Higher Education, 17(5), 633-651. https:// doi.org/10.1108/IJSHE-01-2015-0004

Zhou, G. (2015) Environmental Pedagogical Content Knowledge: A Conceptual Framework for Teacher Knowledge and Development. En S. Stratton, R. Hagevik, A. Feldman, \& M. Bloom (Eds.), Educating Science Teachers for Sustainability (pp. 185203). ASTE Series in Science Education. Springer, Cham. https://doi.org/10.1007/9783-319-16411-3_11 